\title{
Racismo y discriminación laboral: afrodescendientes en Quito
}

\author{
Lydia Andrés ${ }^{1}$
}

\begin{abstract}
Resumen
ESTE ARTÍ́CULO ES UNA APROXIMACIÓN A LA RELACIÓN ENTRE RACISMO Y DISCRIMINACIÓN LABORAL ENTRE LA POBLACIÓN AFRODESCENDIENTE RESIDENTE EN QUITO. SE BASA EN REVISIÓN DE LA BIBLIOGRAFÍA EXISTENTE, FUENTES CUANTITATIVAS Y CUALITATIVAS DE SEGUNDA MANO, ENTREVISTAS Y UN GRUPO FOCAL LLEVADOS A CABO EN QUITO EN EL AÑo 2009. A PARTIR DE UNA DISCUSIÓN CONCEPTUAL ACERCA DE LO LABORAL Y EL RACISMO, DONDE SE DEFINE EL MARCO CONCEPTUAL DE PARTIDA, SE ANALIZAN LOS DIFERENTES ÁMBITOS DE LA DISCRIMINACIÓN LABORAL ENTRE LA POBLACIÓN AFRODESCENDIENTE RESIDENTE EN QUITO: EN EL EMPLEO, LO SALARIAL, LO OCUPACIONAL; Y SE TRATA BREVEMENTE EL TEMA DE LA ESCLAVITUD DOMÉSTICA.
\end{abstract}

Palabras claVe: racismo-discriminación-empleo-afrodescendientes.

\section{Abstract}

THIS ARTICle IS AN APPROACH TO THE RELATIONSHIP BETWEEN RACISM AND JOB DISCRIMINATION AMONG PEOPLE OF African DESCENT LIVING IN QUITO. IT IS BASED ON LITERATURE REVIEW, QUANTITATIVE AND QUALITATIVE SOURCes OF SECOND-HAND, INTERVIEWS AND A FOCUS GROUP HELD IN QUITO IN 2009. From A CONCEPTUAL DISCUSSION ABOUT THE LABOR AND RACISM, WHICH DEFINES THE STARTING FRAMEWORK, WE ANALYZE THE DIFFERENT ASPECTS OF WORKPLACE DISCRIMINATION BETWEEN PEOPLE OF AfRICAN DESCENT LIVING IN QUITO IN EMPLOYMENT, WAGES, THE OCCUPATIONAL ISSUES AND BRIEFLY DISCUSSES THE SUBJECT OF DOMESTIC SLAVERY.

KEYWORDS: RACISM-DISCRIMINATION-EMPLOYMENT-AFRICAN DESCENT.

1 Licenciada en Antropología, PUCE. 


\section{Introducción}

l querer escribir acerca de racismo y empleo, lo primero fue buscar lo que se ha
escrito sobre el tema, aunque no fuera algo específico pero que al menos tocara la
relación entre la discriminación étnica y las formas y condiciones de empleo. Ahí surgió la primera observación que quiero apuntar: no hay estudios sobre la relación entre racismo y desempleo, ni entre racismo y empleo. Si existen textos, no han sido publicados o han sido poco difundidos. Esto no es sorprendente. En un país que es evidentemente racista tampoco se encuentra mucha bibliografía sobre este tipo de discriminación, en base a la idea de raza, y la que existe es reciente.

En términos de empleo y sus variables, desempleo, subempleo, empleo informal, en Ecuador, las estadísticas de organismos gubernamentales, del Instituto Nacional de Estadísticas y Censos del Ecuador (INEC), del Sistema Integrado de Indicadores Sociales del Ecuador (SIISE), toman en cuenta variables como edad, género, nivel de formación, medio rural o urbano, pero la variable étnica no aparece hasta los últimos años. Siguiendo a Peter Wade (2000 [1999]), podemos pensar que en el caso de la población afrodescendiente, esta escasez de datos se enmarca en la invisibilidad de la que han sido objeto en toda Latinoamérica, en la historia enseñada, en la literatura y en las ciencias sociales y humanas.

$\mathrm{Y}$ es que una de las primeras relaciones en que se puede pensar al abordar el tema, es que el racismo sea la causa de las diferencias en las tasas de desempleo según etnia. Pero esta relación no es establecida por los estudios estadísticos realizados en el país, hasta hace pocos años. Por ejemplo, en la publicación Mercado laboral ecuatoriano - Análisis 1990-2005 (STDS, SIISE, 2006), aunque se menciona la variable étnica no se profundiza en su análisis, y al momento de abordar el tema de la discriminación en el ámbito laboral encontramos un apartado sobre la discriminación de género más no sobre la discriminación étnica (Ibíd.: 74). En general, es difícil encontrar estadísticas sobre la discriminación laboral según etnia, y aún más para la población afroecuatoriana. La dificultad que entraña medir la discriminación laboral es significativa pero no justifica la falta de información.

Este trabajo no pretende ser un estudio detallado de la relación entre discriminación laboral y racismo, sino un avance o un estudio preliminar. Además, se limita el tema a la población afrodescendiente asentada en Quito. La población afroecuatoriana y la población indígena han sido históricamente y hasta la fecha las poblaciones más discriminadas del país y las que más han sufrido de racismo. La población afrodescendiente del Ecuador se destaca además por tener las tasas de desempleo más importantes del país -estando éstas asociadas con muy altas tasas de pobreza-, por ser en su mayoría urbanas y por disponer de menor cantidad y calidad de datos sobre su población, tanto en cuanto a lo sociodemográfico como a lo sociocultural. Estos son los elementos que nos hacen centrarnos en este segmento de la población ecuatoriana.

Los avances que se presentan a continuación se basan en la revisión de la bibliografía existente sobre el tema, y también en entrevistas y un grupo focal realizado con el apoyo de una fundación afro de Quito que intenta hacer conocer y difundir la cultura afroecuatoriana y afro en general.

\section{La discriminación laboral}

La discriminación laboral puede ser entendida desde varios enfoques. Un primer enfoque es el jurídico, el de los Derechos Humanos y la ciudadanía. En segundo lugar podemos citar el enfoque económico que se centra en el empleo y sus variables, en el mercado y su funcionamiento. Un tercer enfoque se da desde la sociología, que habla de exclusión. Presento estos tres enfoques a continuación, y termino este apartado refiriéndome al enfoque desde el cual se plantea este análisis.

El enfoque de la ciudadanía y los Derechos Humanos pone énfasis en la igualdad de derechos 
de todas las personas, incluyendo el acceso a servicios y a oportunidades. Según esta perspectiva, la discriminación laboral sería un delito adviniendo en contra del principio de igualdad de los seres humanos.

Las leyes ecuatorianas reconocen la existencia de este tipo de discriminación. Y, varios convenios internacionales ratificados por el Ecuador también se refieren a ésta. En primer lugar se debe destacar que, de manera general, la Constitución de 2008 reconoce la igualdad de todas las personas en materia de derechos, libertades y, algo importante, oportunidades:

\begin{abstract}
“Todas las personas son iguales y gozarán de los mismos derechos, deberes y oportunidades. Nadie podrá ser discriminado por razones de etnia, lugar de nacimiento, edad, sexo, identidad de género, identidad cultural, estado civil, idioma, religión, ideología, filiación política, pasado judicial, condición socio-económica, condición migratoria, orientación sexual, estado de salud, portar VIH, discapacidad, diferencia física;” (Constitución, artículo 11, numeral 2: 2008).
\end{abstract}

Efectivamente, la igualdad defendida en nuestra Constitución implica que nadie puede ser discriminado. Esto es también afirmado y ratificado en convenios internacionales que el Ecuador ha suscrito, así como en el Código del Trabajo. Tres convenios internacionales tienen particular importancia en este tema: los convenios 100, 111 y 169 de la OIT. El convenio 100, de 1951, ratificado posteriormente por el Ecuador, se refiere a la igualdad de remuneración. El 111, de 1958, también ratificado más tarde, se refiere a la discriminación en el empleo y la remuneración. Finalmente, el más reciente, el convenio 169, de 1989 y ratificado por el Ecuador en 1998, se refiere a los derechos de los pueblos indígenas y tribales en países independientes.

Pero, en el caso ecuatoriano, mientras se condena y prohíbe la discriminación laboral y se firman convenios en este sentido, "no existen normas ni procedimientos que permitan a los individuos sujetos de discriminación reclamar un trato igualitario para acceder a las mismas oportunidades” (Azanza, 2004: 2). Aún más, “el Derecho permite discriminaciones legítimas, cuando estas discriminaciones tienen justificación objetiva y razonable” (Vinueza, 2006: 21). Este planteamiento del Derecho ecuatoriano puede ser interpretado de muy diversas formas, sobre todo considerando que no existen mecanismos jurídicos para defender el derecho a la no discriminación.

En cuanto al enfoque económico, se encuentran varios argumentos para explicar las tasas de desempleo, sin que se tome en cuenta de manera seria el que las tasas de desempleo se concentren en grupos humanos particulares y que se distinguen étnicamente. El desempleo, el subempleo, empleo informal, son entendidos como resultado de una crisis económica, del sistema capitalista, de la inserción de los países latinoamericanos en el mercado capitalista, teniendo como consecuencias cambios en la estructura del empleo, precarización del trabajo.

Una de las explicaciones más recurrentes en este sentido se refiere a la crisis de la década de 1980 en América Latina. Según Rendón y Salas (2002 [2000]: 558), dicha crisis “aumentó el desequilibrio del mercado de trabajo, ya que la capacidad de generación de empleos asalariados se vio seriamente mermada”. Esto tuvo como consecuencia el deterioro de las condiciones de trabajo y sobre todo la informalización y precarización del trabajo. En los años 1990, la economía se reactiva, pero a pesar de ello, no disminuyen estos procesos de informalización y precarización. Las necesidades del mercado cambian. Ya no se requiere de la misma mano de obra y gran parte de ella queda relegada sin poder acceder a los empleos formales que pueden estar disponibles y que el mercado ofrece.

Por su parte, para los defensores de la Teoría de la Dependencia América Latina se integró al mercado capitalista en una posición subordinada a causa de la carga histórica de la colonización. A causa de "las particularidades de una industrialización e integración económica dependientes, los sectores capitalistas modernos de los países latinoamericanos no son capaces de integrar toda la fuerza de trabajo desarraigada y sobrante del sector tradicional” (Pries, 2002 [2000]: 525). Esto explicaría que una parte importante de la mano de obra se emplee en el sector informal 
urbano de manera precaria. Así, se trata de algo estructural y no de una situación coyuntural causada por una crisis en un momento histórico particular.

El hecho es que en América Latina y en Ecuador, el desempleo, el empleo precario y el empleo informal son una realidad para gran parte de la población. Según el PREALC, entre 1950 y 1980 “el desempleo abierto se redujo mucho” (Ibíd.: 525), y en mismo tiempo, "entre 1950 y 1990 en toda América Latina el llamado sector informal crece a un ritmo de más del doble (120\%) que el llamado sector formal (50\%). Esta tendencia sigue marcando la década de los noventa” (Ibíd.: 526). Si el desempleo baja, pero el sector informal crece a un ritmo mucho mayor que el empleo formal, es fácil concluir que se da un proceso de informalización y de precarización del trabajo.

Pero, ¿por qué es tal o tal grupo el que se ve más afectado por estos fenómenos económicos? Una respuesta posible es que no se trata de grupos étnicos que se ven más afectados, sino que son de manera general las poblaciones que migran a las ciudades, donde se concentra el trabajo precario y el trabajo informal. Efectivamente, se puede argumentar que si las poblaciones indígenas y afrodescendientes son más afectadas, es como consecuencia de un proceso de migración interna. "Más que una exclusión duradera, existe un proceso de integración de los migrantes sucesivo y en etapas. Se insertan sobre todo en empleos de baja calificación del sector secundario y en actividades de servicio orientadas al sector productivo" (Ibíd.: 530). Y, en el caso ecuatoriano, el periodo sí coincide con procesos de migración interna, aunque afecta principalmente a la población indígena que sale de las haciendas después de las reformas agrarias.

Otra explicación posible es postulada desde la sociología del trabajo. Se trata de entender la discriminación laboral como parte de la exclusión social. Inicialmente, en Europa, se definió el concepto de exclusión social como: "los mecanismos a través de los cuales personas y grupos son despojados de la participación y titularidad de los derechos sociales, o como un proceso que excluye a una parte de la población del disfrute de las oportunidades económicas y sociales” (Gacitúa y Shelton, 2000: 13). Tiene tres dimensiones: política, económica y cultural. Así, la discriminación laboral en base a la adscripción étnica o a ideas de raza sería parte de la exclusión social en su dimensión económica. Es importante recalcar que se entiende que la exclusión social se da a través de mecanismos institucionales y procesos "que determinan que ciertos sectores sociales no tengan las mismas oportunidades de integración que otros” (Ibíd.: 15). Se puede objetar a este enfoque que la noción de procesos es demasiado amplia y vaga, y en cuanto a los mecanismos institucionales, no son suficientes para dar cuenta de la discriminación laboral hacia grupos culturales como los afroecuatorianos y afroecuatorianas, como lo detallaremos posteriormente.

Siguiendo con la noción de exclusión social, Figueroa (2000)menciona una relación interesante. Existe una relación entre desigualdad en la distribución de los ingresos y heterogeneidad social. Es decir: la distribución de los ingresos es más desigual en los países donde hay más diversidad cultural (Ibíd.). Aunque no mencione las poblaciones afrodescendientes -eso es muy común en la literatura de las ciencias sociales en Latinoamérica-, es interesante que el autor vincule estas dos variables. Esta relación entre desigualdad en la distribución de ingresos y heterogeneidad social apoya la idea de que las desigualdades en el ingreso se deben a consecuencias de la colonización, específicamente a la discriminación, la estratificación de grupos culturales, la diferente valoración de culturas y fenotipos, el racismo. De hecho, menciona también que la exclusión de la que han sido sujetas estas poblaciones explicaría "el por qué la expansión del capitalismo en Latinoamérica no ha hecho la gran transformación del trabajo campesino en trabajo asalariado" (Ibíd.: 40). Claro que no se menciona discriminación ni racismo, que son, como lo explicaré a continuación, el trasfondo de la exclusión. Además, tampoco se explica cómo esta exclusión ha sido responsable de la no transformación del trabajo campesino en trabajo asalariado. 
Finalmente, faltan explicaciones menos generales y más reales. Y, como lo menciona Ocampo (2004: 39), "la gran desigualdad social no es una característica del actual modelo de desarrollo. Por el contrario, es un elemento estructural asociado a graves problemas de estratificación social que se han venido transmitiendo de modelo en modelo y de generación en generación”. ¿Cuáles son estos "graves problemas de estratificación social”? Y, ¿Cómo se transmiten “de modelo en modelo y de generación en generación”? La respuesta parece ser el racismo que se configuró durante la Colonia y que perdura, con cambios, hasta la fecha, planteamiento defendido aquí.

Dos autores que han tratado el tema de empleo y discriminación para Latinoamérica, Martha Rangel y Andrés Bello, afirman en este sentido que "la segregación por raza o etnia es, de manera general, uno de los factores responsables de la concentración de estos grupos [afrodescendientes e indígenas] en los empleos de baja calificación, remuneración y prestigio social” (Bello y Rangel, 2000: 61).

\section{Racismo}

La noción de racismo a la que me refiero es un tipo de discriminación que se basa en la idea de raza, entendida ésta como un constructo social; también es un racismo cultural y negado. Es la discriminación en base a una idea -llamada raza- que reúne a seres humanos en grupos que son percibidos como definidos por características negativas y pensadas como innatas o inmutables.

Se entiende el racismo como una ideología, es decir un conjunto de representaciones que se encuentran y actúa en imaginarios y prácticas, y que están presentes en toda la sociedad. Por lo tanto, las relaciones que se establecen entre sus miembros estarán siempre en mayor o menor medida definidas por esta ideología que les permite situarse con relación al otro. El contenido de esta ideología es un producto de la evolución de la sociedad y de las relaciones entre sus miembros.

Desde su origen en la época colonial, el racismo en el Ecuador ha tenido cambios importantes, pero su origen es claro en los contenidos actuales. El color de la piel y cualquier signo visible que pueda ser asociado con un origen indígena o afro-descendiente puede ser suficiente para ser objeto de discriminación. También se mantiene la sobrevaloración de lo blanco y de lo europeo, como ideales que se construyen de la misma manera: color de piel y otros rasgos visibles, no sólo fenotípicos, asociados con características culturales, morales y psicológicas consideradas positivas.

¿Cuáles son las prácticas y los discursos que alimentan los imaginarios y perpetúan este tipo de discriminación? Una primera respuesta es el racismo institucionalizado, el que excluye a ciertos sectores de la población de la entrada a ciertos medios. En segundo lugar están los errores, los estereotipos, el racialismo, el paternalismo, que encontramos a veces de manera explícita y a veces implícita, que pasan por discursos, por representaciones y por comportamientos (Andrés, 2008).

Un aspecto importante de este racismo es su relación con la noción de clase, producto de su evolución histórica. Esto también es planteado por Bello y Rangel (2002: 40):

“Aparentemente el origen étnico-racial influye de manera importante en la posición que ocupan las personas dentro de la estructura social, siendo la discriminación y la exclusión los mecanismos a través de los cuales un grupo dominante mantiene y justifica la subordinación social y económica de otros, reproduciendo y perpetuando la inequidad”. 
Existe efectivamente una relación fundamental entre clase y etnia, la cual queda clara en el contexto del racismo descrito. Dicha relación es producto de la manera cómo se dio y evolucionó el racismo en Ecuador. Y, así como la etnia ha “otorgado” lugares en la estratificación de clase, también ha otorgado ocupaciones, salarios y diferentes prestigios.

Entender el racismo como una ideología producto de una historia, transmitida a través de prácticas, de forma institucionalizada, a través de la endoculturación, en imaginarios, es decir, entendiendo el racismo como un hecho social y cultural amplio, podemos entender la discriminación laboral étnica como una manifestación de este racismo. Al igual que otras manifestaciones de éste, este tipo de discriminación se alimenta y alimenta a su vez al racismo.

En el caso de la población afroecuatoriana de Quito, es importante tomar en cuenta el contexto en el cual se insertan en el mercado laboral, cómo llegan a la ciudad. Al igual que la población indígena, y como lo plantean Bello y Rangel (2000: 1) para Latinoamérica, “desde hace décadas se han visto forzados a emigrar a los grandes centros urbanos donde acceden a trabajos precarios, mal remunerados y de baja calidad”.

\section{Discriminación laboral}

\section{Prejuicios y estereotipos}

Primero es necesario interesarnos un momento en los prejuicios y estereotipos usualmente ligados a la población afrodescendiente en Ecuador. Estos prejuicios y estereotipos son el nivel psicológico del racismo, su base cognitiva. Están presentes en toda la sociedad transmitiéndose de generación en generación en los imaginarios. Son aprendidos generalmente de manera inconsciente desde la infancia. De forma sintética, los prejuicios son ideas preconcebidas, es decir basadas en poco o nulo conocimiento del objeto del prejuicio, y aquí son aplicados a grupos sociales.

Los estereotipos son representaciones simplificadas y generalizadas a un grupo y se refieren en este caso a cualidades y actitudes psicológicas, físicas y morales. El estereotipo es en gran medida producto de nuestra limitada capacidad cognitiva que obliga a almacenar información categorizando y por lo tanto simplificando: es más económico, cognitivamente, almacenar unos cuantos rasgos psicológicos, morales y físicos para describir toda una población, que serán juntados por un elemento en común simple y fácilmente perceptible, que almacenar información sobre cada miembro del grupo referido. El prejuicio está relacionado con él, puesto que muchos estereotipos se basan en prejuicios. Es decir que no solo se basan en poca información sino que pueden basarse en información que no tiene sustento.

Muchos prejuicios y estereotipos han sido ligados a la población afrodescendiente, y estos, al ser parte de la ideología racista, se encuentran en todos los ámbitos de la vida en sociedad, incluyendo el ámbito laboral. Estos pueden o no manifestarse, estar más o menos presentes en los imaginarios de las personas que trabajan junto con afrodescendientes. Veremos aquí algunos de estos estereotipos y prejuicios.

Aunque existen prejuicios y estereotipos racistas comunes a todos los afrodescendientes, algunos son diferentes según el género. Por ejemplo, de manera general existe el estereotipo de la suciedad, detallado por Carlos de la Torre (2002), aunque éste es más frecuentemente asociado a la población indígena. Otro estereotipo es el que asocia a la población afrodescendiente con la sexualidad y lo práctico en general, disociándolos al mismo tiempo de todo lo que se refiere a la intelectualidad. Estos estereotipos y prejuicios influyen claramente en la discriminación laboral.

En lo que concierne a los hombres, estos son asociados con trabajos manuales, con la fuerza física, con la violencia y con la bravura. Esto tiene como consecuencia que se empleen frecuentemente como cargadores y guardias de seguridad (Ibíd.). Los estereotipos son muy 
bien puestos en evidencia por Jean Rahier (1998) en su análisis de las representaciones de afroecuatorianos y afroecuatorianas en la revista Vistazo. Además, estos estereotipos son enseñados y aprendidos en este mismo proceso de discriminación, ya que al no ser contratados en otro tipo de trabajo, quedan asociados a labores manuales, a fuerza física y violencia, $\mathrm{y}$, como la afirma De la Torre (2002: 49): "las construcciones dominantes del hombre negro como esencialmente violento y sólo apto para las labores físicas naturaliza las consecuencias de la esclavitud".

Además, estos prejuicios también inciden en las oportunidades de ascenso. Se puede contratar un afroecuatoriano para un trabajo manual o que implique fuerza física, o se lo puede contratar para una tarea que no implique toma de decisiones. Pero, no tendrá más oportunidad de ascenso, aún si decide prepararse estudiando. Encontramos un ejemplo de ello en un testimonio recolectado por Paloma Fernández (2001: 146): "Parece que todavía la gente, en algunas empresas hay eso de que no le admiten al negro para ocupar más que todo un puesto alto. Si es que le dan el trabajo pero para que siempre está si es posible cargando cajas, como que en ese sentido no quieren que se supere, que llegue a ser un negro jefe de la empresa”.

No sólo hay falta de oportunidades de ascenso, ni exclusión de trabajos profesionales, no sólo se conduce a empleos mal remunerados, difíciles, peligrosos, sin seguridades laborales y de bajo estatus, sino que los estereotipos y prejuicios pueden empujar al desempleo. Efectivamente, también existe el estereotipo según el cual los afrodescendientes son vagos, dejados y lascivos, los cuales son suficientes para ser rechazados al momento de solicitar empleo. De hecho, como lo anota Jhon Antón (2005) a partir de una encuesta sobre racismo, 53\% de los entrevistados dijo que indígenas y afro-descendientes no aprovechan las oportunidades, y el 21\% que son vagos. Es evidente que con estos prejuicios es difícil que se dé empleo a una persona afrodescendiente, y es aún peor si consideramos el estereotipo de "negro ladrón”.

Las mujeres afroecuatorianas, por su lado, como consecuencia de los estereotipos generales que pesan sobre esta población, han sido asociadas al trabajo doméstico -como empleo que no requiere formación profesional ni intelectualidad- y a la prostitución. Esta idea es confirmada por varios autores y testimonios. Por ejemplo, el testimonio de una mujer afroecuatoriana recopilado en las entrevistas publicadas por Andrés Carrión como La memoria de los Otros (1996): “No olvidemos que hay gente que cree que las negras somos prostitutas potenciales porque, por ejemplo, cuando la gente de la sierra va a Esmeraldas, los hombres van con el único propósito de divertirse y luego acostarse con una negra” (Carrión, 1996: 343).

Este tipo de prejuicios puede llevar a casos de agresiones, maltratos, violencias y abusos en el ámbito laboral teniendo como objeto la sexualidad de mujeres afroecuatorianas. Desde la colonia este estereotipo llevaba a que mujeres afroecuatorianas que eran explotadas en el servicio doméstico fueran violadas por sus “dueños” o por los amigos de éste como algo común (Fernández, 2001). No cabe duda de que este tipo de abusos han seguido, aunque en las últimas décadas haya disminuido.

\section{Discriminación ocupacional}

Estos prejuicios y estereotipos tienen mucha incidencia en el ámbito laboral. Otro aspecto que debe ser mencionado que influye en la discriminación laboral son las redes sociales. Las personas entrevistadas y los testimonios recogidos por otros autores coinciden en la importancia que tienen las redes al momento de insertarse en el mercado laboral. Por ejemplo, muchas, si no todas, las mujeres que llegan a trabajar en Quito en el servicio doméstico, lo hacen gracias al contacto de un familiar o conocido que está trabajando en la ciudad. Estas redes ayudan al mantenimiento de la población afrodescendiente en ciertas ramas de actividad, puesto que se mueven en ámbitos de empleo limitados y los empleos conseguidos para familiares y conocidos se encuentran en estos mismos ámbitos. 
Estas ramas de actividad o ámbitos laborales han sido definidas por el racismo y la evolución de éste desde la colonia hasta nuestros días. Esto es lo que se llama discriminación ocupacional, parte de la discriminación laboral. En su estudio sobre discriminación y empleo en Guatemala, Bastos y Camus (1998: 67) afirman que: “existen unas fronteras simbólicas que delimitan los espacios laborales a los cuales los indígenas no acceden. En el caso ecuatoriano encontramos lo mismo, y a ello se debe añadir que los afrodescendientes sufren de la misma discriminación. Estas "fronteras simbólicas" se han definido por el racismo. Producto de ello, encontramos que grupos humanos categorizados con la idea de raza son estigmatizados para realizar sólo cierto tipo de actividades: discriminación ocupacional racista.

Un mecanismo fundamental de la discriminación ocupacional es la discriminación que se da en el acceso al empleo. Un puesto de trabajo no será dado a una persona afrodescendiente con la misma facilidad que a otra persona que no sea afroecuatoriana. Un filtro muchas veces usado y que actúa de manera implícita como mecanismo racista se encuentra en los requisitos para postular a un empleo. Éste es el de "buena presencia”. Requerir "buena presencia” es algo evidentemente vago, subjetivo y cultural. En Ecuador, "buena presencia” implica muchas veces tener un color de piel claro. Además de que puede usarse como excusa para poder negar un puesto a cualquier persona que sea afroecuatoriana -o indígena-, sin necesidad de dar mayor explicación -ya que es un criterio subjetivo-, muchas veces se entiende por "buena presencia" ser considerado bonito/a. Claro, la noción de belleza está también atravesada por el racismo. En un país racista y con nuestra historia, ser bonito/a es casi siempre tener la piel clara, el cabello y los ojos claros. La blancura está asociada con lo bueno, lo bonito y la riqueza, mientras que se asocia la negritud con lo feo, lo malo y lo pobre².

Un testimonio ejemplifica muy bien este tipo de discriminación, aunque en este caso, por un hecho fortuito, el acto racista no tuvo lugar en el momento de la selección. En las oficinas de una exportadora de rosas, una mujer afroecuatoriana, abogada, fue contratada, a pesar de sus estudios y título universitario, como secretaria. Su jefe inmediato aprovechaba las competencias de esta mujer, puesto que aunque su estatus y salario fuera de secretaria, sus tareas incluían actividades relacionadas con los estudios que realizó, de Derecho. Después de un tiempo trabajando en esta empresa, un día, llegó a visitar las oficinas el dueño de la empresa. Éste no conocía a la mujer mencionada. Al verla, no dijo nada. Pero, enseguida, tomando a parte al jefe inmediato de esta mujer, le dijo que la despidiera. Éste le preguntó por qué, con qué excusa. El dueño contestó que le dijera que "no corresponde a la imagen de la empresa". Efectivamente, la mujer fue despedida. Los empleados y empleadas de la empresa que la conocían, todos los que trabajaban en estas oficinas sabían que "fue despedida por negra".

Este testimonio me fue relatado por una empleada de dicha empresa, que funciona en Quito. Aquí se ve claramente la discriminación en el empleo. En primer lugar, la mujer fue despedida por motivos claramente racistas, sin ninguna otra justificación, y con la excusa mencionada en el párrafo anterior: la imagen, o "buena presencia”. Es tan absurda la justificación, aún más sabiendo que esta persona no trabajaba atendiendo a público, que se hace obvio el racismo que causó el despido. Y es que lo que también puede pasar es que en este caso el dueño, quien pide el despido, argumente, negando su racismo, que no es por criterio suyo sino por los gustos y criterios de la gente que es atendida. Pero en este caso, ni siquiera esta justificación racista solapada puede ser argumentada. En segundo lugar, y adelantando un poco sobre las diferentes manifestaciones del racismo en el ámbito laboral, no se da el salario ni el estatus merecido a la mujer afroecuatoriana de esta empresa. Ellos no son acordes ni con su profesión, puesto que es secretaria cuando se formó como abogada, ni con sus actividades, puesto que no sólo no se reconocen sus estudios sino que no se reconoce su labor trabajando como secretaria y como abogada.

2 Véase el estudio de Andrés (2008) donde se evidencia cómo estas imágenes racistas están presentes en imaginarios de niños y niñas, y cómo se construyen. 
Con esto podemos pasar a otra manifestación de la discriminación ocupacional. Ésta también se refiere al tipo de empleos a los que acceden los afrodescendientes. Retomando lo que se dijo acerca de la educación y las transformaciones en las economías y el mercado del trabajo, es interesante notar que "dentro del sector moderno los afroecuatorianos y los indígenas son quienes menor participación tienen (35,9\% y 24.2\% respectivamente)", frente a 49\% y 43,11\% para blancos y mestizos, respectivamente (SIISE, 2004: 62). Aunque el sector moderno está definido de manera bastante amplia, éste incluye ramas de actividad que requieren educación superior. Ésta es sin duda una de las razones por las cuales tanto indígenas como afroecuatorianos tiene menor participación. Además de la discriminación a nivel de educación, como causa de esta diferencia también se puede incluir la discriminación en el acceso al empleo y casos de discriminación en el empleo como el que se acaba de mencionar.

De manera más específica quiero volver a mencionar las diferencias de género. En el estudio de paloma Fernández, se menciona el tipo de actividades en que las mujeres afroecuatorianas se dedican en Quito, esto desde los años 1990:

"la mayoría de las mujeres que conocí no tenían empleo formal remunerado, habían llegado a trabajar en el servicio doméstico o se dedicaban a otras actividades de la economía informal como la venta ambulante, el lavado de ropa y en menor medida el servicio sexual. Algunas mujeres gestionaban pequeños negocios de venta en su domicilio, las menos eran empleadas en medianas y grandes empresas; y también en instituciones públicas como las guarderías del barrio, y el centro de salud del Ministerio.” (Fernández, 2001)

La inserción en trabajos que no requieren educación superior o profesionalización es clara. Esto a pesar que un porcentaje no desdeñable de ellas tienen educación superior.

Además de las ramas de ocupación, también se debe mencionar el trabajo precario, subempleo y el trabajo informal. Las definiciones se superponen en varios aspectos, teniendo en común la falta de seguridad y de estabilidad en el empleo. Por ejemplo, como lo explica Pries (2002), el trabajo informal puede ser definido según la legalidad de las actividades o la seguridad social, o según el nivel de ingresos o de estabilidad, o según el tipo de ocupación. El subempleo y el trabajo precario entran también en estas definiciones.

En el caso de la población afroecuatoriana en Quito, en una publicación del SIISE, se menciona que "en las zonas urbanas el fenómeno del subempleo afroecuatoriano está asociado a actividades de ventas callejeras, servicio doméstico mal remunerado y servicio de carga en los puertos” (SIISE, 2004: 62-63). Así, en cuanto a ocupaciones, los afroecuatorianos y afroecuatorianas están mayoritariamente empleados sin estabilidad y sin seguridad, además de ser frecuentemente mal pagados. En cuanto al seguro social, indicador de seguridad, "la población afroecuatoriana registra el promedio más bajo con apenas el $21,1 \%$, incluso un poco por debajo de la tasa de la población indígena (21.8\%)", mientras que los "blancos" tienen 37,4\% y los mestizos 33,3\%" (SIISE, 2004: 65).

\section{Discriminación salarial}

Otro tipo de discriminación es la que se da en salarios. "La salarización formal, una característica propia del proceso modernizador, queda fuera del alcance de los grupos que recién se instalan en la ciudad” (Bello y Rangel, 2000: 21). Esto incluye a los afroecuatorianos y afroecuatorianas que se instalan en Quito, y se ve acentuado por la discriminación ocupacional de la que son objeto. Como vimos, la población afroecuatoriana no tiene el mismo acceso al sector moderno que la población "blanca” y mestiza, y se encuentra por lo tanto en gran medida excluida del tipo de salarización formal. Es así relegada a un tipo de trabajo precario con menos seguridades laborales y con más posibilidad de ser mal remunerada. 
Por otro lado, también se pueden apreciar diferencias de ingresos que no son atribuibles únicamente a diferencias de formación o competencia. En una publicación del 2007, de la OIT, sobre la discriminación en el mundo, se encuentran datos interesantes, que a pesar de ser generales sí son aplicables al Ecuador. Se presenta el salario mediano por hora de mujeres afrodescendientes, hombres afrodescendientes y mujeres "blancas" como porcentajes con relación a los hombres "blancos”, los cuales tienen salarios más elevados. Aunque estos datos corresponden a Brasil, es interesante anotarlos, puesto que se presentan como ejemplares. Los salarios más cercanos al de los hombres "blancos" es el de las mujeres "blancas", seguidas de los hombres afrodescendientes y finalmente las mujeres afrodescendientes (OIT, 2007: 28). Es decir que en lo referente a salarios la discriminación racialista tienen más influencia que la discriminación sexista, y que al conjugarse las dos, las mujeres afrodescendientes son las personas más discriminadas.

Además de la falta de contratos y su consecuente falta de salarización formal, y además de las diferencias en salarios con relación a la población "blanca” y mestiza, existen otras consecuencias de la discriminación en cuanto a ingresos que se dan en el hecho de ser retribuido en especies, total o parcialmente, y en los retrasos en las remuneraciones (OIT, 2007: 33). Por ejemplo, es una práctica común el considerar como parte del sueldo el dar almuerzo a empleados que trabajan sin contrato formal. Otra práctica relacionada es dar posada y considerarlo como una manera de pagar el trabajo realizado. En las entrevistas realizadas se pudo confirmar que esto ocurría en los años 1960 y 1970, y aunque es una práctica que ha disminuido, aún existe, sobre todo en el ámbito del servicio doméstico que detallaremos más adelante.

\section{Discriminación en el empleo}

En el empleo pueden darse varias formas de discriminación racista. Una de ellas ya fue mencionada: se trata de la falta de reconocimiento. Este tipo de discriminación puede estar asociado a otro que se refiere a las oportunidades de ascenso, que también fue mencionado. En estos dos casos se puede hablar de racismo en el sentido en que la falta de reconocimiento del trabajo o de la formación, y la falta de oportunidades de ascenso, no tienen que ver con requerimientos del trabajo y no pueden ser justificados de ninguna manera que se relacione con las tareas a ser cumplidas.

Por otro lado, lo que también puede tener lugar en los lugares de trabajo son comportamientos racistas, como chistes, insultos, malos tratos y también paternalismo, forma solapada de racismo. De manera general, este tipo de comportamientos racistas son frecuentes, en todos los ámbitos. Pero, es posible que este tipo de comportamiento hay disminuido, tal vez gracias a las manifestaciones en contra del racismo y al mayor reconocimiento de la plurietnicidad en el país y a las luchas de movimientos indígenas y afroecuatorianos. En los años 1960, los que llegaron a Quito, sufrieron "ataques insultantes de manera sistemática” (Fernández, 2001: 141). En entrevistas realizadas en el año 2009 se pudo constatar que los ataques son sistemáticos, pero también parece que son menos frecuentes en la generación más joven. Desafortunadamente, no existen datos estadísticos sobre este tema que permitan ver su evolución. Un aspecto interesante es que el contenido de estos ataques no ha cambiado mucho. Para los años 1960, "entre los insultos más frecuentes se encuentran alusiones al color de modo muy despectivo y en forma de metáforas asociadazas a la nocturnidad, la suciedad, el mal agüero y el diablo” (Fernández, 2001: 141). A esta lista se podría quitar las alusiones al diablo y aumentar alusiones a la vagancia. 


\section{Esclavitud doméstica y trabajo infantil}

Finalmente, quiero mencionar una consecuencia de la colonización y de la esclavitud que se ha justificado dentro de la ideología del racismo y que no termina de desaparecer: la esclavitud doméstica. Con respecto a este tema, Bello y Rangel, refiriéndose al caso de Brasil y algunos países del Caribe, mencionan "esclavitud informal”: "sociedades que después de la abolición transitan de una esclavitud formal a un sistema de dominación o "esclavitud informal” aún más complejo que el anterior” (Bello y Rangel, 2002: 43). Esto no es explicado por los autores que se limitan a mencionarlo brevemente. Aunque la situación debe ser diferente, es por la historia de colonización común que se pueden aproximar los casos.

En Ecuador la esclavitud no desapareció en el momento en que se emitió la ley de manumisión de esclavos a mediados del siglo XIX. Además de la tardanza en aplicar la ley y contando que los imaginarios no cambian rápidamente y aún menos de manera determinista con relación a la emisión de una ley, la esclavitud dejó de existir para dar paso al huasipungo para la población afroecuatoriana. El sistema de endeudamiento al que fueron encausados, a pesar de ser una mejora, no significó libertad.

Como vimos, a partir de los años 1960 empieza la migración a Quito -y otros centros urbanos. Muchas de las mujeres son niñas o adolescentes que llegan para trabajar en el servicio doméstico a través de redes sociales. En otros casos también hay niñas que son traídas a Quito por familias "blancas" o mestizas. Este fue el caso de una mujer cuyo testimonio se encuentra en Fernández (2001). Es el caso de una mujer que fue "robada", traída a los cuatro años de edad de Esmeraldas a Quito para servir a sus "patrones":

\footnotetext{
"Yo me críe en esa casa [de servicio] hasta... yo le tuve a mi hija a los 17 años, de ahí yo me salí, porque mucho me pegaban, y a cada rato. El trato que a uno le dan de niño, se puede decir, debe de ser con cariño y no maltratar. Muy mal trato, siempre-siempre fue así. Tal es que yo no tenía nombre. Nunca me llamaban por mi nombre: tal es que a mí no me hace mella que me digan negra. $\mathrm{Al}$ menos, cuando mi patrona estaba brava, mi nombre era solamente: negra desgraciada, negra infeliz. Ese era mi nombre: negra esto, negra el otro...” (Ibíd.: 148)
}

Este es el testimonio de una mujer afroecuatoriana que tenía en los años 1990, 64 años. Los malos tratos, la explotación y esclavización que sufrió esta mujer sólo se pueden justificar por la ideología racista.

En el 2009, una mujer afroecuatoriana de Imbabura, de aproximadamente 65 años, me relató una historia parecida. Ella vino a Quito siendo niña, por un contacto de un familiar que ya trabajaba en la ciudad. Llegó directamente a una casa donde sirvió de empleada doméstica hasta el momento en que se casó. Durante esos años, ella vivió en la casa de sus "patrones", quienes la "dejaron ir a la escuela”, le dieron ropa y un lugar donde dormir. No se le dio ningún tipo de remuneración. Logró acabar la escuela, pero no pudo ir al colegio. Siendo adolescente, aproximadamente a los 16 años de edad, empezaron a darle algo de plata, pero no como remuneración sino como un regalo para que pudiera salir de vez en cuando. Varios aspectos deben ser rescatados de este testimonio. Primero, la actitud de esta señora de profundo agradecimiento hacia sus "patrones" porque no la trataron mal. Segundo, la ausencia de remuneración y contrato. Tercero, la falta de acceso a la educación. Cuarto, la falta de libertad. 
Este tipo de hechos de esclavitud ha cambiado. Aunque todavía hay mujeres afroecuatorianas que viven esta situación en años posteriores y seguramente todavía existe, ahora es más común que las mujeres tengan algún tipo de remuneración, pero sin seguridad social, sin salarización ni contratación formal, y con parte de la remuneración en especies (habitación, comida, ropa). Por ejemplo, en el 2007 una adolescente de 15 años, afroecuatoriana, me contó cómo llegó a Quito para trabajar en el servicio doméstico en una casa de clase media en el norte de Quito. Ella no fue “negociada” entre gente "blanca” de Quito y su madre o padre, sino que llegó a trabajar por redes familiares. Esta mujer no tenía contrato, ni seguridad social. Su salario era muy bajo, y además era víctima de malos tratos. Respondiendo al prejuicio de fechoría, aunque ella tuviera un cuarto -si se lo puede llamar así- y viviera en la casa, no se le entregaban las llaves de la casa, de tal manera que podía quedarse un día entero fuera de la casa esperando a que llegaran los dueños. Es así que pude conversar con ella, ya que en esa ocasión, los dueños de casa habían salido en la mañana en un momento en que ella no estaba, y ellos no regresarían hasta el final del día. También relató un problema al que tenía que enfrentarse con los dueños de casa y que ejemplifica la sobrevivencia de las relaciones de esclavitud en los imaginarios. Para no pagarla, siendo el primer mes que ella trabajaba allí, le habían reclamado diciendo que había usado el teléfono para hacer llamadas a otras provincias y que eso cubría lo que debían pagarle. Ella manifestó que era falso que ella hubiera hecho llamadas y que ese reclamo lo hacían para no pagar lo que debían. Ya que este tipo de trabajo se realiza sin contrato, ella no disponía de ningún mecanismo para reclamar lo que le debían, su remuneración. Claramente, los dueños de casa no debían pensar que era necesario pagarla por su trabajo, reviviendo la esclavitud de siglos pasados.

Muchas de las afroecuatorianas que llegan a trabajar en el servicio doméstico son adolescentes, y en otros casos son mujeres que vienen con niños y/o niñas pequeños. La regulación ecuatoriana indica que hasta los 15 años de edad se sigue hablando de trabajo infantil. A partir de 16 años el trabajo no es considerado como en contra de los derechos de los niños, niñas y adolescentes, pero tiene una legislación especial. Las tasas de trabajo infantil son un claro indicador de la discriminación que existe hacia poblaciones indígenas y afroecuatorianas. Efectivamente, estas poblaciones tienen más altos índices de trabajo infantil. Por un lado, esto es el resultado de la discriminación laboral de la que son sujetos sus padres y madres, que los obliga en muchos casos, como estrategia de reproducción del hogar, a hacer trabajar a sus hijos e hijas menores, sin lo cual no podrían subvenir a las necesidades del grupo familiar. Por otro lado, estos niños y niñas se ven afectados en diferentes aspectos y en su acceso a la educación y su rendimiento escolar. Así, de manera indirecta, el trabajo al que muchos niños y niñas indígenas y afrodescendientes se ven obligados por necesidades económicas los perjudica a largo plazo en sus posibilidades de acceso al mercado laboral, en lo que se refiere a salarios y ocupaciones sobre todo.

\section{Conclusiones}

Hemos visto que la discriminación laboral hacia afroecuatorianos y afroecuatorianas en Quito es una manifestación más del racismo que existe en el país. Éste es el producto de la historia colonial del Ecuador y de su evolución durante la República. En este contexto, etnia y clase han evolucionado como categorías coextensivas. Ser afrodescendiente en Ecuador ha sido sinónimo de ser pobre. El racismo ha determinado que no accedan de la misma manera a los recursos económicos, a la educación, a los servicios que debería proporcionar el Estado que sólo recientemente ha empezado a asumir su rol con la población afroecuatoriana.

Dentro del contexto racista del país, ha sido coherente que la población afroecuatoriana sea discriminada en el empleo, en el acceso a éste, en las oportunidades de ascenso, en lo ocupacional. Y, como en cualquier otro ámbito de la vida en sociedad, los afroecuatorianos y afroecuatorianas han sufrido ataques racistas en sus empleos. Los prejuicios y estereotipos racistas fraguados durante la Colonia no han cambiado mucho y siguen siendo parte de los 
imaginarios de ecuatorianos y ecuatorianas. Estos, además son reforzados y transmitidos en los comportamientos y actos racistas.

Los estereotipos y prejuicios de vagancia, fechoría, violencia, sexualidad exacerbada, falta de intelectualidad, han afectado de manera decisiva la inserción en el ámbito laboral. Así, afrodescendientes han sido relegados a empleos de bajo prestigio, sin seguridad social, mal remunerados, sin contratación ni salarización formal, empleos manuales y que no impliquen decisión ni intelectualidad. Además, la esclavitud arraigada en los imaginarios ha tenido consecuencias nefastas haciendo posible que niñas y adolescentes afroecuatorianas sean convertidas en esclavas domésticas.

Pero podemos también mencionar aspectos positivos. Los cambios en las últimas décadas, logrados por la presión y manifestación de movimientos de reivindicación étnica y de lucha contra el racismo, en Ecuador y en el mundo, han tenido consecuencias positivas. Se ha tomado consciencia de la discriminación y ha sido denunciada, aunque lamentablemente los reconocimientos provenientes del Estado no han dado paso a la implementación de mecanismos jurídicos para luchar eficientemente contra la discriminación. Estos vacíos hacen posible que la discriminación producto del racismo pueda seguir siendo parte de la vida cotidiana de muchos y muchas en el país de manera solapada y negada en la mayoría de los casos y muchas veces de forma explícita.

$\mathrm{Y}$ es que los reconocimientos son un avance pero no son suficientes. Se necesita que los imaginarios cambien. Pero, estos, producto de la cultura, no cambian rápidamente. El racismo se transmite de generación en generación. Las condiciones de discriminación no permiten que padres y madres ofrezcan a sus hijos e hijas los recursos para poder salir de estas condiciones de pobreza, exclusión y discriminación.

Vivir en una sociedad capitalista implica que existan explotados. La noción de raza sirvió durante siglos para justificarla. Si se la deja de lado, con seguridad se encontrará otra u otras para justificar la explotación de unos por otros. El racismo llamado cultural, por ejemplo, es una muestra de ello: si no se puede usar la noción de raza ya caduca, se puede hablar de diferencias culturales que funcionen como la noción de raza.

Finalmente, quiero apuntar ciertos aspectos de la relación entre discriminación laboral y racismo que no fueron abordados o apenas mencionados y que merecen ser profundizados: trabajo infantil, población indígena, extranjeros y xenofobia, género y etnia. Por otro lado, también sería necesario discutir la propuesta de la discriminación positiva e interesarse por los casos exitosos de inserción en el mercado laboral por miembros de grupos discriminados. 


\section{Bibliografía}

Andrés, Lydia. 2008. Imaginarios en formación - aprendiendo a pensar al otro en un colegio de élite de Quito. Ecuador: Abya Yala.

Antón S., Jhon. 2005. “Introducción” en: Secretaría Técnica del Frente Social, Sistema de Indicadores del Pueblo Afroecuatoriano -SISPAE- Racismo y discriminación racial en el Ecuador. Ecuador: República del Ecuador, Ministerio de Bienestar Social.

Azanza, M. 2004. Delineamientos jurídicos tendientes a una mayor eficacia de la no-discriminación laboral en la legislación ecuatoriana. Tesis de licenciatura, Pontificia Universidad Católica del Ecuador.

Bastos, Santiago y Manuela Camus. 1998. La exclusión y el desafío: estudios sobre segregación étnica y empleo en ciudad de Guatemala. Guatemala: FLACSO.

Bello, Álvaro y Marta Rangel. 2002. "La equidad y la exclusión de los pueblos indígenas y afrodescendientes en América Latina y el Caribe”; en: Revista de la CEPAL, n. 76, Abril 2002. México: CEPAL. 39 - 54

Bello, Álvaro y Marta Rangel. 2000. Etnicidad, “raza” y equidad en América Latina y el Caribe. Chile: CEPAL.

Carrión, Andrés. 1996. La memoria de los Otros. Ecuador: Eskeletra editorial.

Centro de Estudios de Población y Desarrollo Social. 1985. Inmigración a Quito. Ecuador: Centro de Estudios de Población y Desarrollo Social.

De la Torre, Carlos. 2002. Afroquiteños: ciudadanía y racismo. Ecuador: Centro Andino de Acción Popular.

Fernández R., Paloma. 2001. Diáspora africana en América Latina: discontinuidad racial y maternidad política en Ecuador. España: Servicio Editorial de la Universidad del País Vasco.

Figueroa, Adolfo. 2000. “La exclusión social como una teoría de la distribución” en: Estanislao Gacitúa, Carlos Sojo y Davis Shelton H. ed. Exclusión social y reducción de la pobreza en América Latina y el Caribe. Costa Rica: FLACSO, Banco Mundial.

Gacitúa, Estanislao y Davis Shelton H. 2000. Introducción - Pobreza y exclusión social en América latina y el Caribe en: Estanislao Gacitúa, Carlos Sojo y Davis Shelton H. ed. Exclusión social y reducción de la pobreza en América Latina y el Caribe. Costa Rica: FLACSO, Banco Mundial.

Ocampo, José. 2004 “Desarrollo Económico e inclusión social”; en: Mayra Buvinic ed., Inclusión social y desarrollo económico en América Latina; Colombia: BID.

Oficina Internacional del Trabajo. 2007. La igualdad en el trabajo: afrontar los retos que se plantean. Suiza: OIT.

Oficina Internacional del Trabajo. 1989. Convenio 169: Convenio sobre pueblos indígenas y tribales en países independientes. Suiza: OIT.

Pries, Ludger. 2002 (2000). “Teoría sociológica del mercado de trabajo” en: De la Garza Toledo, Enrique coord., Tratado latinoamericano de sociología del trabajo. México: Colegio de México, FLACSO, Universidad Autónoma Metropolitana, Fondo de Cultura Económica.

Rahier, Jean. 1998. “Representaciones de gente negra en la revista Vistazo, 1957-1991”. Iconos 7, pp. 96-105.

Rendón, Teresa y Carlos Salas (2002 (2000)) “El cambio en la estructura de la fuerza de trabajo en América Latina” en: De la Garza Toledo, Enrique coord., Tratado latinoamericano de sociología del trabajo. México: Colegio de México, FLACSO, Universidad Autónoma Metropolitana, Fondo de Cultura Económica.

Secretaría Técnica del Ministerio de Desarrollo Social, Sistema Integrado de Indicadores Sociales del Ecuador. 2006. Desempleo en el Ecuador. Ecuador: Secretaría Técnica del Ministerio de Desarrollo Social.

Secretaría Técnica del Ministerio de Desarrollo Social, Sistema Integrado de Indicadores Sociales del Ecuador, Sistema Integrado de Indicadores Sociales de la Población Afroecuatoriana. 2004. Los afroecuatorianos en cifras - Desigualdad, discriminación y exclusión según las estadísticas sociales del Ecuador. Ecuador: Secretaría Técnica del Ministerio de Desarrollo Social.

Vinueza, M. 2006. La tutela del derecho a la igualdad y no discriminación de actos u omisiones provenientes de particulares. Tesis de licenciatura, Pontificia Universidad Católica del Ecuador.

Wade, Peter. 2000 (1999). Raza y etnicidad en Latinoamérica. Ecuador: Abya-Yala. 